\title{
Problems of pressure estimation in high temperature experiments using solid media apparatus with particular reference to piston-cylinder and anvil-with-hole apparatus (Part I)
}

\author{
ROBERT A ISHBULATOV and ALEXANDER V KOSYAKOV \\ Institute of Experimental Mineralogy, USSR Academy of Sciences, 142432 Chernogolovka \\ Moscow District, USSR
}

\begin{abstract}
The piston-cylinder apparatus has been widely used in geochemical experiments for more than 25 years, but no accurate technique to measure pressures in conjunction with high temperatures has so far been developed. One should however, take into account many possible sources of errors in pressure measurements in case of a piston-cylinder apparatus.

The anvil-with-hole type (AH) high-pressure apparatus is less popular, although it might be successfully employed to carry out experiments at pressures up to $100 \mathrm{~kb}$. In-situ technique for the AH-23M type of apparatus (anvil-with-hole, $23 \mathrm{~mm}$ in cell diameter) deals with the measurement of pressure in each run at room temperature by using $\mathrm{Bi}-\mathrm{Tl}$ pressure gauges. There is uniform pressure distribution within the working part of the cell at room and high temperatures. The temperature distribution is also uniform (within $5^{\circ} \mathrm{C}$ ) at high pressure. The equipment operates with two-stage compression. The use of these methodical approaches makes it possible to accurately perform experiments at pressures from 25 to $50 \mathrm{~kb}$ and high temperatures (to $1600^{\circ} \mathrm{C}$ ) with accuracy.
\end{abstract}

Keywords. Pressure estimation; high temperature experiments; solid-media apparatus; piston-cylinder; anvil-with-hole apparatus.

\section{Introduction}

Most experimental studies at pressures above $10 \mathrm{~kb}$ were conducted by using the piston-cylinder apparatus (Boyd and England 1960). Although this apparatus was used successfully for 25 years, no conventional technique has so far been developed to calibrate P-T condition with precision. Anvil-with-hole apparatus (AH) is less popular (Sirota et al 1974), but has an advantage over the piston-cylinder apparatus in that experiments with this equipment are possible not only in the pressure range of 25 to $50 \mathrm{~kb}$, but also up to $100 \mathrm{~kb}$.

In routine experiments there are at least two parameters (e.g. pressure and temperature of equilibration), which need to be measured accurately. Precise pressure measurement is a difficult problem because both types of equipment work on the same principle, and solid media are used for transmitting pressure. The P-T calibration is of great importance because direct comparison of results of investigation made in different laboratories is possible.

The pressure exerted on a sample by a piston in the piston-cylinder apparatus is computed as force per unit area. The calculation takes into account empirically determined corrections. They include (i) losses due to friction between the ram and the walls of a hydraulic cylinder $\left(\Delta F_{1}\right)$; (ii) friction between the cell, and the walls of the chamber $\left(\Delta F_{2}\right)$, which in turn takes into account the friction between the moving 
piston (or rather the locking ring adjacent to the piston face) and the chamber wall; (iii) friction losses and other possible effects within the cell arising from inherent rheological properties of its material. $\Delta P_{3}$ is the correction related to nonuniform distribution of pressure (gradient) along the cell, whereas $\Delta P_{4}$ pertains to corrections for the nonuniform distribution of pressure in the cross-section of the cell (the socalled anvil effect which may be positive or negative (Bell et al 1971; Ishbulatov 1977). A correction for variations in the cell cross-section area, $S_{\mathrm{ch}}$ (an increase in diameter of the chamber at high pressures) is denoted as $\Delta P_{5}$.

The losses, $\Delta F_{1}$ and $\Delta F_{2}$, may be related to $S_{\mathrm{ch}}$ and defined as $\Delta P_{1}$ and $\Delta P_{2}$, i.e.

$$
\begin{aligned}
& \Delta P_{1}=\Delta F_{1} / S_{\mathrm{ch}} ; \\
& \Delta P_{2}=\Delta F_{2} / S_{\mathrm{ch}} .
\end{aligned}
$$

In in-stroke runs, the 'real pressure' on the sample $\left(P_{r}\right)$ at room temperature may be written as:

$$
P_{r}=P_{0}^{\text {in }}-\Delta P_{1}-\Delta P_{2}-\Delta P_{3} \pm \Delta P_{4}-\Delta P_{5},
$$

where $P_{0}$ is the "nominal pressure" computed with the equation:

$$
P_{0}=P_{\mathrm{oil}} \cdot S_{\mathrm{ch}} / S_{\mathrm{ch}},
$$

where $P_{\text {oil }}$ is the oil pressure in the hydraulic cylinder and $S_{\text {ch }}$ its cross-sectional area. The sign of $\Delta P_{3}$ in piston-in runs will always be negative, because the pressure at the bottom of the cell is higher than that in the middle. The sign of $\Delta P_{4}$ depends on compressibilities of material in the central and peripheral parts of the cell: if the column of material which encloses the sample is more compressible than its surroundings, then $\Delta P_{4}$ is negative (a negative anvil effect). In other words, in this case the pressure in the innermost part of the cell (with the sample) is lower than that in its outermost part and $\Delta P_{4}$ is positive (a positive anvil effect) under inverse circumstances. The negative sign of $\Delta P_{5}$ reflects expansion of the chamber at high pressures.

In out-stroke runs, equation (1) is rearranged as follows:

$$
P_{r}=P_{0}^{\text {out }}+\Delta P_{1}+\Delta P_{2}+\Delta P_{3} \pm \Delta P_{4}-\Delta P_{5}
$$

The change in the sign of $\Delta P_{3}$ is related to the appearance of an inverse pressure gradient: pressure in the middle of the cell is higher than that at its bottom. $\Delta P_{4}$ has essentially the same sign in both in- and cut-stroke runs, since the correction applied is solely caused by rheologic properties of the cell material. Thus, the above equations show that measurement of the 'real equilibrium pressure' even at room temperature presents some problems.

There are several ways to solve the problems. One correction may be determined experimentally $\left(\Delta P_{1}\right)$, the second may be allowed if they are the same in both in- and out-stroke runs $\left(\Delta P_{2}\right)$, whereas the third may be minimized provided the cells are made of appropriate material $\left(\Delta P_{3}, \Delta P_{4}\right)$.

The correction $\Delta P_{1}$ may be determined provided the press is calibrated utilizing a forcemeter, or it might be eliminated entirely if the forcemeter is employed for nominal pressure measurements throughout the run (Johannes 1978). The use of grease-base $\mathrm{MoS}_{2}$ suspension and a lead foil jacket minimized $\Delta P_{2}$ is most likely the 
same in piston-in and piston-out runs. The magnitude $\Delta P_{3}$ may be diminished if the cell assembly is made of material of lower internal friction. However, it still remains obscure whether these corrections are of identical magnitude in in-stroke and out-stroke runs.

In early experiments with a model pyrophyllite cell $(12.5 \mathrm{~mm}$ in diameter and $45 \mathrm{~mm}$ in height) two Bi-sensors (located in the middle and at the bottom of the cell) indicated a marked difference in pressure at around $25 \mathrm{~kb}$ for both piston-in and piston-out runs. The difference is in the range of $1.5 \pm 0.2 \mathrm{~kb}$ and about $0.2 \mathrm{~kb}$ for in-stroke and out-stroke runs, respectively (lshbulatov 1978). In the case of similar $\mathrm{NaCl}$ cells, the differences in pressure measurements are $0.8 \pm 0.2 \mathrm{~kb}$ and about $0.2 \mathrm{~kb}$ for in-stroke and out-stroke runs respectively (unpublished data).

In case the cell assembly is made up of the same material, the magnitude $\Delta P_{4}$ is most likely to be zero. It is difficult to determine experimentally, the $\Delta P_{5}$ value which, however, may be negligibly small (presumably up to $40 \mathrm{~kb}$ ) provided a WC core is used.

Nevertheless, we could make simple calculations for illustration. Let the WC core be of 12.7 and $50 \mathrm{~mm}$ diameters (inner and outer respectively) i.e. its wall thickness is $18.5 \mathrm{~mm}$. If at $30 \mathrm{~kb}$ the bore diameter is assumed to increase by $0.07 \mathrm{~mm}$ (this corresponds to $0.2 \%$ decrease in wall thickness provided the outer diameter is constant), then it will yield a $1 \%$ error in pressure calculations which amounts to $0.3 \mathrm{~kb}$.

Thus it follows from the above discussion that the 'real equilibrium pressure' at room temperature may, at best, be calculated from in- and out-stroke determinations with the following equation:

$$
P_{r}=\frac{P_{0}^{\text {in }}+P_{0}^{\text {out }}}{2}-\frac{\Delta P_{3}^{\text {in }}-\Delta P_{3}^{\text {out }}}{2}-\left( \pm \Delta P_{4}+P_{5}\right)
$$

If the corrections applied are unknown, then the second and third terms of the equation will correspond to uncertainties in estimating pressure.

Determination of each type of correction in the experiments at room temperature is of academic interest, since in practice it is more advisable to calibrate a pressure device (cell assembly) by using known phase transitions with respect to some "reference" metals $(\mathrm{Hg}, \mathrm{Bi}$, etc) and pressure being measured under hydrostatic condition. Replacing the sample within the cell assembly by a "reference" metal and measuring the nominal pressure at the phase transition suggests that the difference between $P_{0}$ and $P_{r}\left(P_{r}\right.$ corresponds to the pressure for the phase transition) will amount to the sum of all the corrections applied:

$$
P_{0}-P_{r}= \pm \Sigma \Delta P \text {. }
$$

It is possible that such a calibration yields the magnitude $\Delta P$ for the solely-fixed value of nominal (or real) pressures. Therefore, high precision calibration of high-pressure apparatus (cell) over the entire desired pressure range requires a set of sensors like $\mathrm{Hg}, \mathrm{Bi}$ and $\mathrm{Tl}$ ones. Since the latter are regrettably limited in supply, the corresponding calibration procedure is also of restricted possibilities.

Also, there is another similar method to calibrate pressure by using diminutive pressure gauges made up of "reference" metals (e.g., $\mathrm{Bi}, \mathrm{Tl}$; Ishbulatov and Litvin 1975; Ishbulatov 1977). The essential precondition required for the method is that the pressures at the point where the pressure gauge is located and the sample area must be identical. The method, as applied to an AH-23 M (anvil-with-hole) apparatus is further discussed below. 
In high temperature experiments, the procedure of pressure estimation was more complicated than in experiments at room temperature, because in the former case the cell assembly must be of at least two materials which normally include graphite as heater and conventional material for other parts of the cell. Some workers (Mirwald et al 1975; Johannes 1978) employed low-friction $\mathrm{NaCl}$-graphite cells. However, in contrast to runs carried out at room temperature (under isothermal conditions), the nonuniform distribution of temperature in the cell for different runs (i.e. at relatively high and relatively low temperatures) both within and beyond the graphite heater, respectively, will involve changes in rheological properties of $\mathrm{NaCl}$, the transmitting medium. Thus, although the cell material is homogeneous in chemical composition, the cell itself is similar to that made of material of different physical properties. Since the increase in temperature reduces internal friction of the pressure medium, $\mathrm{NaCl}$ cells should be characterized by a negative anvil effect, i.e. the pressures measured will be lower than 'real' ones. In other words, $\mathrm{NaCl}$ cells indicate increase in $\Delta P_{4}$ against a decrease in $\Delta P_{3}$, the former being zero at room temperature. However, it is important to note that heating above certain temperatures causes a pressure rise by a magnitude of $\Delta P_{6}$, owing to thermal expansion in the pressure-transmitting medium. Such a pressure rise will differ in different parts of the cell reflecting a variable temperature distribution. Clearly, the increase in the real (or sample) pressure is not necessarily similar to the increase in the total (integral) pressure in the cell i.e. the increase of the force applied to the piston due to thermal expansion of the cell. If the pressure increase force exceeds the magnitude $\Delta P_{2}$, it will be fixed by a forcemeter. If it exceeds the value of $\Delta P_{2}+\Delta P_{1}$, an increase of the gauge (or nominal) pressure involving a piston displacement will be recognized. In this case, both inand out-stroke cycles, the direction of the piston displacement will be the same, corresponding to that characteristic of out-stroke cycles. Hence, the logic underlying pressure determination (or the magnitude of the pressure corrections needed) based on the results from in- and out-stroke runs seems invalid. The relation between the nominal and sample (real) pressures in high temperature runs (which use cell assemblies made up of material of different rheological properties) may be described theoretically with equations (1) and (3). In this case a proper correction for thermal expansion $\Delta P_{6}$ should be applied. However, it must be remembered that physical properties (internal friction, compressibility etc.) will change to a variable degree with temperature leading to variable values of corrections, $\Delta P_{3}$ and $\Delta P_{4}$. In routine experiments, pressure corrections become complicated, because of the use of different material as pressure-transmitting media. In some cases, heating the cell causes a nominal pressure drop. This is due to processes related to the variation in the volumes of the substance during heating, crystallization of amorphous substances (corundum), softening of pyrex glass at $800^{\circ} \mathrm{C}$, and dehydration of hydrous material such as talc and pyrophyllite. Because of relatively sluggish kinetic processes, allowance should also be made for the time factor involved in the experiments.

A few more cases regarding pressure changes with temperature are considered here. The arguments presented are sufficient to show that the pressure calibration procedure in the piston cylinder high pressure apparatus is a complex problem, indeed.

In such a situation, the most logical solution for the problem will be calibration on the basis of high temperature phase equilibria studies, in an apparatus with a hydrostatic medium (refer Ishbulatov and Kosyakov 1990, in this volume, referred to hereafter as Part II). 
In contrast to the piston-cylinder apparatus the pressure generated in the $\mathrm{AH}-23 \mathrm{M}$ type apparatus cannot be calculated, since it is difficult to determine the area on which the force is applied (see text below). Therefore, the experimental procedure involves calibration of the cell at room temperature by using pressure gauges in each run. The necessary precondition required for the procedure is determination of the relation between the pressure in the gauge and sample pressure, and that between sample pressures at room and high temperatures.

The experimental procedures to solve the above problems and the technical details of the AH-23M apparatus are discussed below.

\section{Anvil-with-hole apparatus (AH-23M)}

The AH-23M apparatus consists of opposed anvils with holes on their faces. The holes are similar to cell assemblies in shape and size, i.e. hemispherical and toroidal holes in the centre and around the periphery (figure 1). A tungsten carbide core is laterally supported by hardened steel rings. Supported plates also contain the WC core, whose diameter is essentially two times greater than that of the anvil. The supported plate and the anvil unit are cooled by water, whose flow rate is controlled and regulated.

As a source of force a 500-ton commercial press is employed, with an additional high pressure oil pump, connected to a hydraulic system. This enables regulation of



Figure 1. Anvil-with-hole apparatus (AH-23M) 1: static ram of press; 2: paper insulated laminated insulator; 3: support plate; 4: WC anvil with semi-spherical hole; 5: steel ring; 6: solid media cell; 7: water cooler; 8: moving ram. 


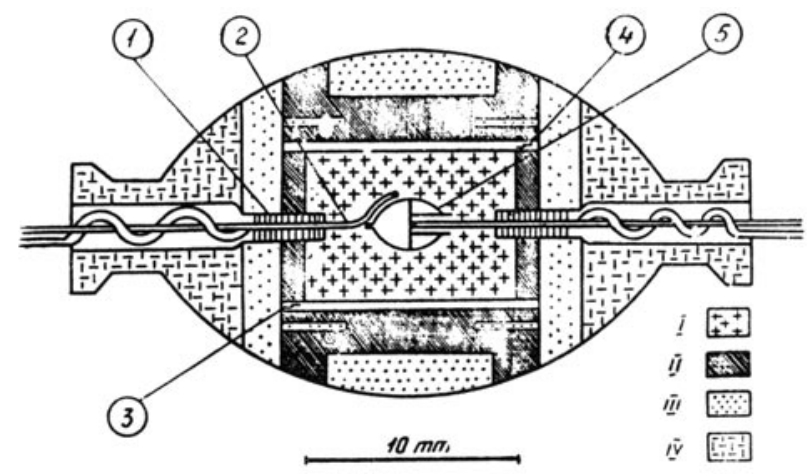

Figure 2. Solid state cell for anvil-with-hole apparatus. 1: alimina tube (insulation for thermocouple wire); 2: thermocouple; 3: pressure sensor (Bi, T); 4: graphite heater; 5: ampule I: boron nitride; II: graphite; III: pyrophyllite; IV: lithographic stone. The single-ampule variant is shown on the left side and the two-ampules variant is shown on the right side.

a ram displacement rate over wide ranges $(0.05$ to $1.0 \mathrm{~mm} / \mathrm{min})$. Oil pressure applied to a hydraulic cylinder was measured with a Bourdon tube gauge. Electrical power was supplied to the graphite heater via anvils, hence the top (static) ram is isolated from the press body by an insulating disc.

The temperature was controlled to $1^{\circ} \mathrm{C}$ using a special electronic system which permits regulation of the input power by a power sensor which converts the power into a signal. The latter approximately corresponds to the e.m.f. of the $\mathbf{P t}_{70} \mathbf{R h}_{\mathbf{3 0}} /$ $\mathrm{Pt}_{94} \mathrm{Rh}_{6}$ thermocouple to measure the temperature within the cell.

The cell of this apparatus (figure 2) is made up of limestone. The sleeve made up of unfired pyrophyllite, is placed between the body and the graphite heater. It keeps limestone from melting in the runs at high temperature $\left(>1200^{\circ} \mathrm{C}\right)$. It was previously found (Ishbulatov 1977) that unless the pyrophyllite sleeve was used, the melting of such material produced a marked increase in the real pressure (up to $60 \%$ at $1500^{\circ} \mathrm{C}$ ) which was difficult to control.

The major components of the graphite furnace are disc-shaped covers with wire inlet and a sleeve. A furnace construction of this type has an advantage over a tube because the geometry and size variations of different components make it possible to set up virtually any configuration related to the temperature field inside the furnace including the uniform temperature distribution. The inner space of the furnace (working space) is filled with two cylindrical parts made up of boron nitride which have hemispherical holes on their faces for capsules with samples. Capsules are made up of either $\mathrm{Pt}$ or $\mathrm{Pt}_{60} \mathrm{Rh}_{40}$ alloy. There are two horizontal channels in the cell (usually oriented at an angle of $60^{\circ}$ to one another) for thermocouple out-let.

The cell is seen to crack at the very beginning of the compression but no considerable changes occur in its geometry. With increasing applied force outer shell material creeps into the space between the anvils until the gap becomes indistinguishable. Cell material then practically stops moving in and pressure inside the cell begins to increase.

The apparatus reportedly allows pressures up to $50 \mathrm{~kb}$, to be reached; the material properties of the anvils place an upper limit on the pressure range. 


\subsection{Temperature and pressure measurements}

The temperature was measured with a $\mathrm{Pt}_{70} \mathrm{Rh}_{30}-\mathrm{Pt}_{94} \mathrm{Rh}_{6}$ thermocouple. No correction for the pressure effect on the e.m.f. of the thermocouple was made. The temperature gradient within the sample was tested by a method (Ishbulatov 1977) which assumes that if the temperature is slightly lower than that of the liquidus then the distribution of the crystalline phase in a melt (glass) is chemically homogeneous. This indicates that the temperature distribution is uniform. It is clear that this condition may be realized only at a certain melt viscosity and run duration when the crystallina phase has not settled. We commonly use andesite melts in this kind of experiment. An experiment conducted above the liquidus of an andesite resulted in the formation of homogeneous glass. Another run was carried out at a temperature $10^{\circ} \mathrm{C}$ lower than the liquidus. This run yielded small crystals distributed uniformly throughout the glass. Run products are easy to estimate since in most cases a sample can be wholly taken out of a capsule after opening it. The uniform temperature distribution was achieved by changes in the geometry of the heater. It was found during our investigation that the use of the thermocouple wire of $0.5 \mathrm{~mm}$ diameter (which possesses the necessary mechanical stability) distorts the configuration of the thermal field to a great extent. Therefore, a thermocouple with wires of different diameter was used $(0.3 \mathrm{~mm}$ in the internal part of the cell and $0.5 \mathrm{~mm}$ in its external part). The ends of the wires of different diameters are connected by a spiral (figure 2) which is made of thermocouple wires of the same composition. It is logical that the changes in geometry and size of the heating elements cause the changes in pressure distribution in the working part of the cell. Temperature and pressure distributions were then conducted in successive cycles.

The pressure distribution in the working part of the cell was measured at room temperature using a 'wandering sensor' (Ishbulatov 1977). A method commonly used consists of two pressure gauges placed in the cell. The first one ("reference") has its constant position and the second ("wandering") could be placed in different parts of the cell. Pressure gauges are short pieces $(2-3 \mathrm{~mm})$ of the $\mathrm{Bi}$ and $\mathrm{Tl}$ wire of $0.1 \mathrm{~mm}$ diameter incorporated into the slanting holes in filter paper. The reference gauge was placed between the disc and the cylindrical elements of the heater, whereas the "wandering" one was placed between the two copper foil discs anywhere within the working part of the cell. The successive electric connection of both gauges was ensured. The diagram, reflecting the changes of electric resistance of the chain related to pressure, shows a jump separate jumps in the electrical resistance of the gauges if the difference between the nominal pressures exceeds $0.2 \mathrm{~kb}$.

Experiments show (figure 3 ) that the pressure distribution depends to a large extent on the geometry of the electrical input and that it changes with cyclical loading of the cell. These effects were rather insignificant at $25.5 \mathrm{~kb}\left(\mathrm{Bi}_{\mathrm{I}-\mathrm{II}}\right.$ transition pressure), and pressure gradients inside all the investigated cells were less than $0.25 \mathrm{~kb} / \mathrm{mm}$. However, at $36.7 \mathrm{~kb}\left(\mathrm{Tl}_{11-111}\right.$ transition pressure) the pressure distribution depends to a great extent on the type of electrical input. The maximum pressure gradient for the cell used in the following experiments (figure 2) was $0.2 \mathrm{~kb} / \mathrm{mm}$ at room temperature (pressure 25-50 kb).

Pressure variation within the sample is not greater than $0.3 \mathrm{~kb}$ using the nearspherical capsules of $3 \mathrm{~mm}$ in diameter (at the maximum). In order to investigate the pressure distribution in the cell at high temperature a new method has been used. It 


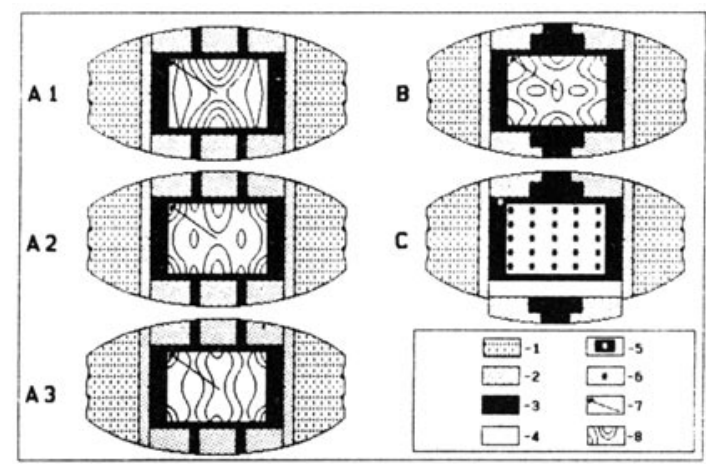

Figure 3. Effect of graphite heater construction under recycle loading on the pressure distribution in the cell of anvil-with-hole apparatus determined with thallium pressure sensors. A1B: first, A2 second, A3: third loading cycles. C: position of "zero" and "floating" pressure sensors. 1: limestone; 2: pyrophyllite; 3: graphite; 4: hexagonal BN; 5: "zero" pressure sensor; 6: "floating" pressure sensor; 7: direction of pressure increasing (end of arrow shows point at $36.7 \mathrm{~kb}) 8$ : isobaric lines through $0.5 \mathrm{~kb}$.

is based on the well known fact that the quartz $\rightleftarrows$ coesite transition above $1000^{\circ} \mathrm{C}$ is characterized by the absence of a hysteresis loop in its P-T path. Both capsules (one with quartz and another with coesite) were inserted simultaneously into the cell during the experiments. In such experiments, capsules were made in the form of hemispheres with flat caps. In order to have a uniform pressure distribution within the working part of the cell during experiments under certain P-T condition, a capsule with quartz and the other with coesite + quartz was used. Naturally in the latter case, the coesite-to-quartz ratio is dependent on run durations. Hence, in the P-T range of stability of coesite we shall have one capsule with coesite and the other with quartz + coesite. If the two phase-assemblages developed are different from one another it suggests nonuniform pressure distribution within the sample, and the pressure range should characterize the pressure difference within the sample.

Experiments on high temperature-pressure distribution were carried out at $1000^{\circ} \mathrm{C}$ at different steps with an interval of $0.5 \mathrm{~kb}$ (refer Table 3 , of part II). When the cell was used (figure 2), the presence of two phase associations in the cell in both capsules was observed only in the experiment at $30 \mathrm{~kb}$. The above considerations confirm that the pressure gradient within the sample does not exceed $0.15 \mathrm{~kb} / \mathrm{mm}$. In similar experiments with another cell (shown in figure $3 a$ ) the pressure gradient within the sample is about $0 \cdot 3-0 \cdot 4 \mathrm{~kb} / \mathrm{mm}$.

One of the most important results from the pressure distribution data is that in order to determine pressure within a sample it is sufficient to measure pressure at the point where the 'reference' gauge is installed. The pressure required at high temperature is set up with the help of the two stage-compression (Ishbulatov 1977), i.e. preliminary cell compression, heating up to a given pressure. The logic underlying this procedure is discussed below.

Let us assume that a homogeneous solid body, eg. sphere (figure 4) is compressed under the action of the applied force $(F)$; the shape of the body is of little importance in further discussion. Because of internal friction in the medium the sphere shows a pressure gradient directed to its surface, i.e. toward the region of higher pressure. The magnitude of pressure difference, $\Delta P$, between the values of pressure on the surface 


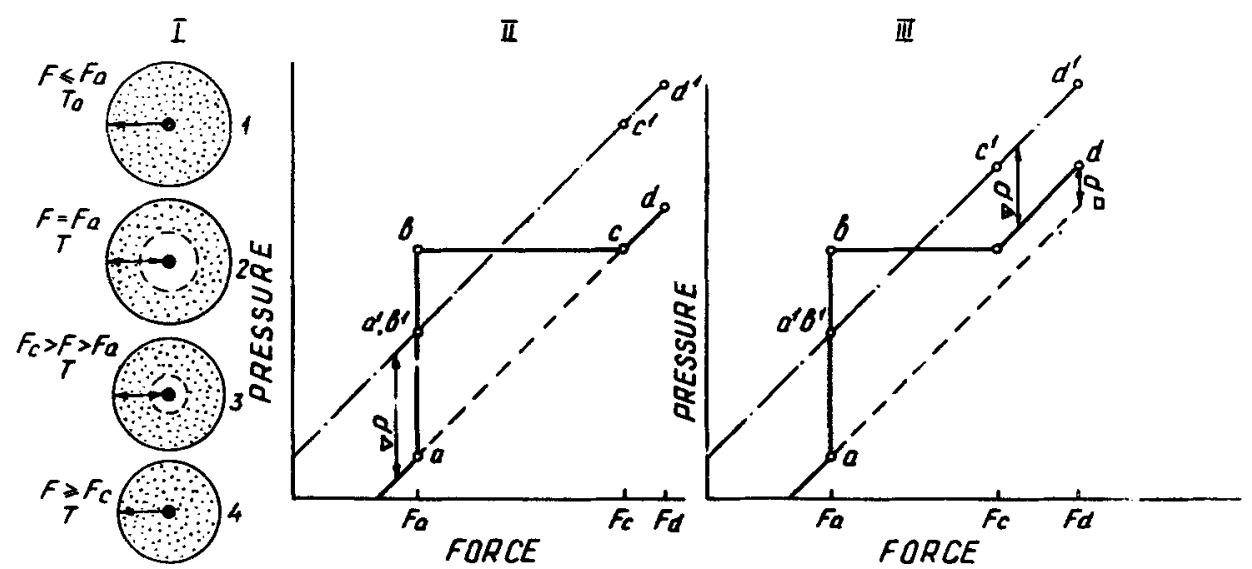

Figure 4. I: Direction of pressure gradient in compressed homogeneous solid state sphere at room $\left(T^{0}\right)$ and high temperature $(T)$. II: force-pressure dependence in centre ( $\boldsymbol{P}_{c}$-solid line) and surface ( $P_{s}$-dashed line) of solid sphere. Rheological properties of temperature from $T_{0}$ to $T . \nabla P=P_{s}-P_{c}$-difference between values of pressure at surface and at the centre of the sphere at room temperature. III: Force-pressure dependence, but sphere material shows change in rheological properties during heating $\nabla P=P_{s}-P_{c}$ at high temperature $T$, $\square P=\nabla P-\nabla P$.

$P_{s}$ and the centre $P_{c}$ may be expressed as

$$
\Delta P=P_{s}-P_{c} .
$$

For clarity, we assume that $\Delta P$ is constant over the entire pressure range (i.e. $P_{s}$ and $P_{c}$ versus $F$ lines are parallel, figure 4 , II). We now suppose that under the action of the force $F_{a}$, the central portion of the sphere is heated up to the required temperature $T$. As a result, $P_{c}$ increases (see a-b in figure 4 , II). A certain amount of force caused by thermal expansion decays again due to internal friction in the medium. Therefore, the inner portion of the heated sphere exhibits an inverse pressure gradient (figure 4, 1-2). It is possible that in this case $P_{s}$ is invariable. If the sphere is first heated up and then compressed, $P_{c}$ will be constant until the pressure gradient is directed toward the surface at any point of the sphere (figure 4, 1-4, see also $F_{a}-F_{c}$ in figure 4, II). If the rheological properties of the medium are invariable during heating at the required temperature $T$, the pressure gradient and, accordingly, the pressure difference will be identical to those in a "cold" compression cycle (at room temperature).

If the force applied is greater than $F_{c}$ (see figure 4 , II), then the force dependence of pressure on the sample in a 'hot' two-stage compression cycle is the same as that in a 'cold' cycle. In that case, the calibration procedure is valid for both 'cold' and 'hot' cycles. However, internal friction in solid media decreases with temperature, resulting in reduction of the pressure gradient. As a consequence, the pressure difference at high temperature $\Delta P$, is less pronounced than that at room temperature (figure 4, III). This implies that the $P$ value determined in a 'cold' compression cycle will be lower $(\square P=\nabla P-\nabla P)$ than the true one at temperature $T$ of interest. In practice, the experimental apparatus used cell assemblies made up of various materials. Therefore, we will now consider a sphere which comprises two media with different 


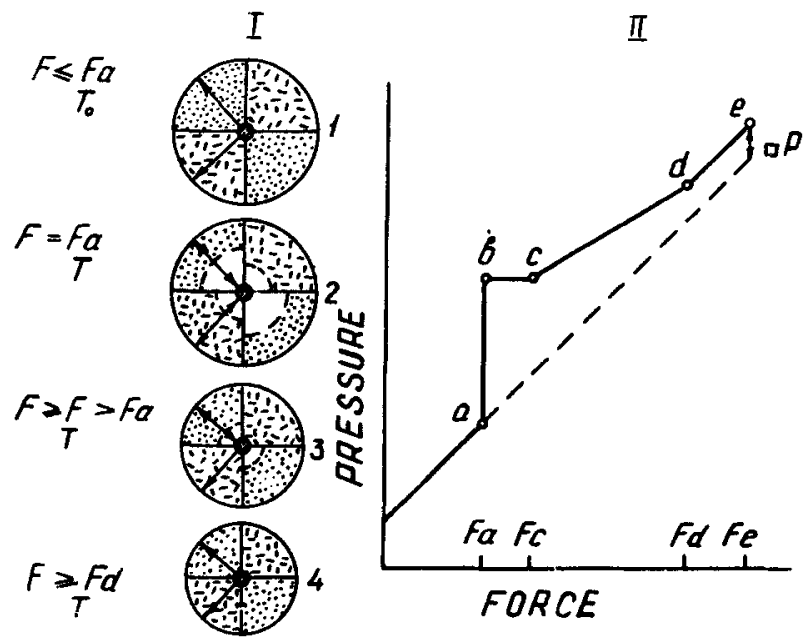

Figure 5. I: Direction of pressure gradient in compressed two-media spheres. II: Forcepressure dependence at the centre of the sphere.

rheologic properties (figure 5). The result of inhomogeneity of the sphere is that each medium exhibits its own pressure gradient both for 'cold' and 'hot' cycles. Thus, when finally compressing the sphere (containing the heater), $\boldsymbol{P}_{c}$ will be invariable until the pressure gradient of any of the media is directed toward the outermost shell of the sphere (figure 5-I, see b-c and in figure 5, II). As only a portion of the sphere (one of the media) is capable of transmitting the force applied, the $\boldsymbol{P}_{c}$ value is markedly lower (see c-d in II of figure 5) than that observed as a result of compression of the 'cold' sphere, with both media being pressure transmitters. A further increase in the force applied is characterized by pressure gradients directed towards the surface at any point of the sphere. Consequent variations in $\boldsymbol{P}_{c}$ may be described with a relationship which differs by the magnitude $\square P$ from that of the 'cold' cycling process. It should also be stressed that this magnitude takes into account the differences in properties of the media when heated to the intended temperature $T$.

Clearly, the above discussion as applied to actual apparatus (e.g., anvil-with-hole, piston-cylinder) will lead to a similar conclusion. In two-stage compression runs, there is no pressure correction caused due to thermal expansion. Furthermore, the pressure exerted on the sample at high temperature and defined by calibration at room temperature differs from the true one. The magnitude of the difference takes into account variation in rheological properties of the pressure media at increasing temperature.

If the run temperature is significantly lower than the temperature of softening (melting) of the pressure-transmitting media, the variation in their rheological properties may be minor, and the $\square P$ value could be neglected. Hence, under these conditions, the calibration procedure at room temperature may be successfully used for pressure determination at elevated temperatures with sufficient accuracy. However, this may be done only provided the magnitude of an interval of 'hot compression' is known, because it is strongly specific for each cell type (e.g., dimensions, material) at each temperature. This magnitude may be determined by assuming that each run is performed at the same pressure, and temperature. One should apply different forces 


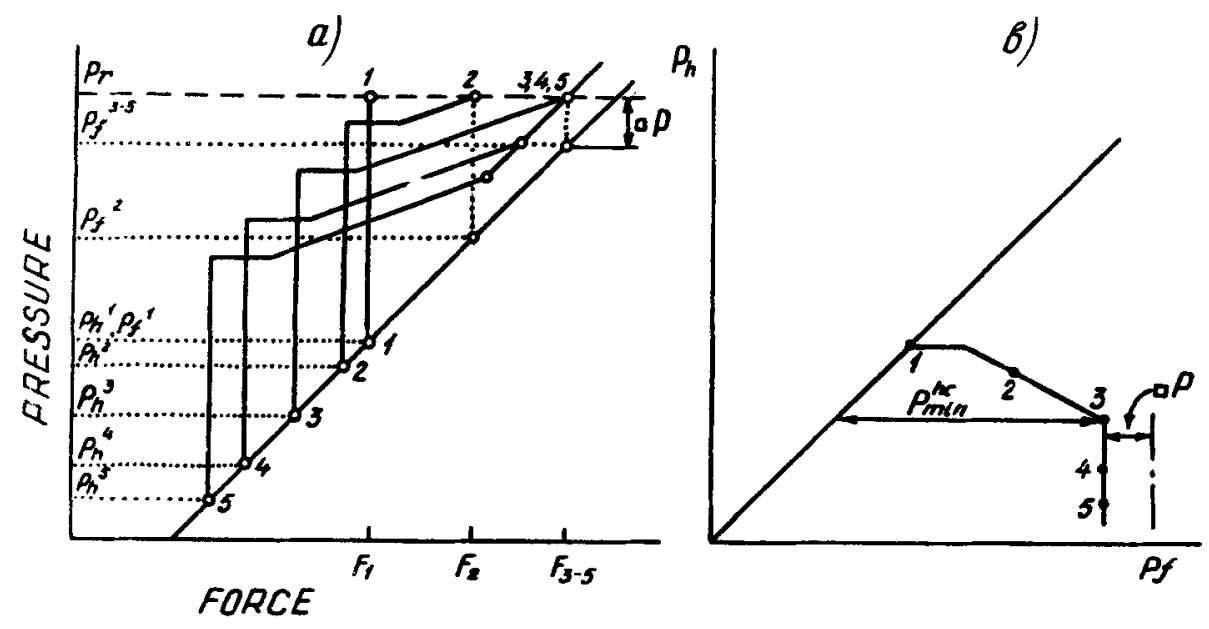

Figure 6. Methodology of determining minimal hot compression interval (see text). $P_{h}$-pressure of heating; $P_{\boldsymbol{f}}$-final pressure.

to achieve $P_{r}$ (reference phase transition). By varying the magnitude of the interval of 'hot compression', the transition pressure can be attained at the expense of the force corresponding to point 1 (figure 6a), and with the increase of thermal pressure (1-1). If heating occurs at a lower pressure (see point 2 in figure 6), then $P_{r}$ can be achieved at the expense of the force corresponding to point 2 , thermal expansion and hot compression'. Figure 6 shows how the pressure related to heating $\left(P_{h}\right)$ and the intended final pressure $\left(P_{f}\right)$ and the pressure exerted on the sample $\left(P_{r}\right)$ is attained. Both pressures were determined from the force versus pressure curve at room temperatures. $P_{r}$ may be derived at the expense of the force corresponding to point 4 . Determination of $P_{r}$ is dependent on the thermal expansion and final 'hot compression'. The final path is a function of pressure and temperature (refer figure 6). At point 3 , if the curve related to temperature versus force (during the two-stage compression procedure) cuts the high-temperature dependence line then $P_{r}$ can be determined. Note that $P_{f}^{3}-P_{h}^{3}$ difference is equal to the minimum magnitude for the interval of hot compression' $\left(\boldsymbol{P}_{\mathrm{min}}^{\mathrm{hr}}\right)$ required to eliminate pressure contribution due to thermal expansion.

The above magnitude can be determined from the $P_{f}$ versus $P_{h}$ diagram at a pressure exerted on the sample $\left(P_{r}\right.$ in our case) at a given temperature (figure $6 \mathrm{~b}$ ). Simple geometric constructions clearly show that $P_{\min }^{h c}=\left(\right.$ ie. $P_{f}^{3}-P_{h}^{3}$ ) will be equal to the distance between point 3 and the line drawn at an angle of $45^{\circ}$ from the origin of coordinates (i.e. the line for which $P_{f}=P_{h}$ ). $P_{r}$ for points 3-5 is attained by applying one and the same force $\left(P_{f}^{3}=P_{f}^{4}=P_{f}^{5}=P_{r}-\square P\right)$. Hence the lines 3-5 in figure 6 correspond to $P_{r}$ (abscissa) measured by calibration at room temperature. This value differs by a magnitude $\square P$ from the true one (figure 6b, dashed line). The magnitude $\square P$ is caused by changes in rheological properties of the pressure-transmitting media during heating. Therefore the use of refractory material permits measurement of pressure at high temperatures with reasonable accuracy on the basis of room temperature calibration.

Detailed studies designed to determine the minimum interval of 'hot compression' were conducted (Ishbulatov 1977) with a similar apparatus (AH-23) employing a cell 
made up of the same material. The quartz-coesite equilibrium curve was investigated to calibrate pressure and temperature. The minimum interval of 'final compression' was found to vary between 6.5 and $7.5 \mathrm{~kb}$ and between 1200 and $1400^{\circ} \mathrm{C}$. Therefore, the present paper is confined to studies of the quartz-coesite equilibrium at $1200^{\circ} \mathrm{C}$.

It appears that the value of 'hot compression' $(7,8$ and $9 \mathrm{~kb}$ ) yields the same value at equilibrium pressure. Taking into account a possible temperature dependence, the minimum interval of 'final compression' is assumed to be $9 \mathrm{~kb}$.

It follows from the previous discussion that the accuracy and reproduction of pressure depend to a great extent on the accuracy of measurements of pressure as a function of force at room temperature. This task is further compounded, because within the pressure range of $20-40 \mathrm{~kb}$, we could use only two values: $\mathrm{Bi}_{\mathrm{I}-\mathrm{II}}=25.5 \mathrm{~kb}$ and $\mathrm{Ti}_{\mathrm{II}-\mathrm{III}}=36.7 \mathrm{~kb}$, i.e. we have to use a method which makes it possible to obtain the pressure dependence within this range.

In the case of the anvil-with-hole apparatus, the pressure was calibrated in three loading cycles up to $40 \mathrm{~kb}$. The values of applied force corresponding to the pressure of phase transitions of $\mathrm{Bi}$ and $\mathrm{Ti}\left(\mathrm{Bi}_{\mathrm{I}-\mathrm{II}}, \mathrm{Tl}_{\mathrm{II}-\mathrm{III}}\right.$ for the second cycle and $\mathrm{Bi}_{\mathrm{I}-\mathrm{II}}$ (for the third cycle) were fixed in the second and third cycles. It has been determined statistically (after conducting 200 experiments) that $S$ and $Z$ values are practically constant. Note the following equations

$$
\begin{aligned}
& S=\mathrm{Bi}_{\mathrm{I}-\mathrm{II}}^{\mathrm{II}}-\mathrm{Bi}_{\mathrm{I}-\mathrm{II}}^{\mathrm{iII}}, \\
& Z=\mathrm{Tl}_{\mathrm{II}-\mathrm{III}}^{\mathrm{II}}-\mathrm{Tl}_{\mathrm{II}-\mathrm{III}}^{\mathrm{III}} .
\end{aligned}
$$

The accuracy of pressure measurement was about $\pm 0 \cdot 15 \mathrm{~kb}$ in the experiments carried out at room temperature (note the real and statistically determined deviations of $S$ and $Z$ values from the constant ones). The necessity to control the cell dimension corresponding to given values with an accuracy of $\pm 0.02 \mathrm{~mm}$ should also be pointed out.

The nonlinear character of the pressure versus force was considered. This dependence has been studied according to the values of the applied force (oil pressure in the cylinder of the hydraulic press) corresponding to the above $\mathrm{Bi}_{\mathrm{I}-{ }_{I I}}$ and $\mathrm{Tl}_{\mathrm{hl}-\mathrm{III}}$ transitions and to the beginning of pressure generation in the working part of the cell. The inflection point on the curve showing the change in the graphite heater resistance marked the beginning of pressure generation. A function of the type $P=a^{*} \ln (F+b)-c$ was used to describe the $P=f(F)$ dependence. The $a, b, c$ values were chosen by a computer.

The above methods enable one to conduct the experiments within the range of 20 to $40 \mathrm{~kb}$ at $1000-1500^{\circ} \mathrm{C}$ to an accuracy of $\pm 1 \mathrm{~kb}$, corrections for changes of the rheological properties of the cell material during heating were not considered. The use of such refractory material as boron nitride, graphite and pyrophyllite allows us to make corrections related to inner changes in the rheological properties. This correction is of negative value and consequently the deduced pressure in this study is slightly greater.

\section{Concluding remarks}

The high pressure solid media apparatus used for pressure transmission, has a remarkable advantage over the hydrostatic devices, especially in the case of high 
temperature experiments because they enable one to approach the elevated pressure during the runs. It is also apparent that the fabrication and use of these devices are much more simpler than the hydrostatic devices. However the technique of precise pressure measurement at high temperature is a complicated problem. Any solid medium high pressure apparatus is characterized by pressure gradients, which are determined by the rheological properties of the material through which pressure is transmitted. As a rule, solid cells are made of various materials. The differences in their compressibility result in the generation of nonuniform pressure distribution within the cell (the so-called anvil effect, Bell et al 1971; Ishbulatov 1977).

The possible increase of the bore diameter in a piston cylinder apparatus under elevated pressures also leads to an uncontrolled error of pressure measurements. In general, the corrections with respect to the nominal pressure in the piston cylinder apparatus are based on the assumption that the corrections for the in-stroke and out-stroke runs are equal, characterized by the opposite signs. However, the corrections, caused by an anvil effect and by the increasing bore diameter possess the same sign, but if one takes into account the lengthwise pressure gradient then the corrections in the out-stroke and in-stroke runs may be different. The corrections relating to the increase of thermal pressure can be considerably decreased if one applies the two-stage compression cycle. However this procedure is useful only when there is an increase in the nominal pressure i.e. if the run temperature surpasses the value of minimal hot compression. It should be noted that some scientists have already used the two-stage compression technique (Johannes et al 1971). In the runs carried out at the relatively low temperatures $\left(600^{\circ} \mathrm{C}\right)$, with respect to the equilibrium reaction albite $\rightleftarrows$ jadeite + quartz, the value of hot compression ( $2 \mathrm{~kb}$; Johannes et al 1971) seems to be reasonable, but with increasing temperature it would be rather insufficient. In order to approach the necessary pressure values under high temperatures within the AH-23M apparatus the authors succeeded in working out a construction of the cell, which favours the uniform pressure distribution within the working part of the cell both at room temperature and at high temperature (pressure gradient $0.15 \mathrm{~kb} / \mathrm{mm}$ ) and provides uniform temperature distribution (within the range of $5^{\circ} \mathrm{C}$ ). The experimental technique presupposes the calibration of a cell during each run at room temperature. The application of hot compression (minimal interval $=9 \mathrm{~kb}$ ) enables one to use this calibration for pressure determinations at high temperature without the correction, attributed to the changes in the rheological properties of the material (used for the construction of the cell) during heating. The use of the refractory material (i.e. boron nitride, graphite, pyrophillite) allows one to assume this value as being insignificant. The accuracy of our pressure measurements $( \pm 1 \mathrm{~kb})$ corresponds to the one assumed for the experiments with piston cylinder apparatus. This correlation seems to be quite reasonable because these corrections for both types of devices are of identical origin. The precision of $\pm 0.2 \mathrm{~kb}$ is therefore believed to be unrealistic. The solution of precise pressure measurement is connected with the development (in future) of the devices with hydrostatic media which will help in the determination of high temperature pressure equilibria (quartz-coesite transformation, for instance).

At present the comparison of the positions of equilibrium lines (albite-jadeite + quartz, quartz-coesite, etc.), obtained by different authors, allows one to determine the range of discrepancy in pressure measurements, though it does not allow one to completely solve the problem of precise pressure measurement. All these questions are considered in the second accompanying paper (Part II). 


\section{Acknowledgement}

The authors are grateful to Prof V A Zharikov for his constant interest in this work and for fruitful discussions. The details of the runs and experimental data were discussed with Dr Yu A Litvin whose remarks were of considerable importance for the authors. F Seifert and Alok K Gupta reviewed an earlier draft of the manuscript and their comments were helpful in the significant improvement of the paper. This paper is dedicated to the late Sir C V Raman, on the occasion of his birth centenary.

\section{References}

Bell P M, Mao H K and England JL 1971 A discussion of pressure distribution in modern solid-pressuremedia apparatus; Carnegie Inst. Washington, Yearb. 70277

Boyd F R and England J L 1960 Apparatus for phase-equilibrium measurements at pressures up to $60 \mathrm{~kb}$ and temperatures up to $1750^{\circ} \mathrm{C} ; J$. Geophys. Res. 65741

Ishbulatov R A 1977 Experimental study on the melting phase relations of calc-alkaline rocks at pressures of 25-45 kb. In Contributions to physical-chemical petrology (Moscow: Nauka Moscow) pp. 96-145 (in Russian)

Ishbulatov R A 1978 Problems of pressure measurements at high temperature in solid-media cells of high-pressure apparatus. In Experimental and techniques of gas and solid-media pressures (Moscow Nauka) pp. 177-184 (in Russian)

Ishbulatov R A and Litvin Yu A 1975 Pressure distribution in cell of solid state apparatus "anvil-with-hole" up to 3 GPa.; Prib. Tekh. Eksp. 3 218-220 (in Russian)

Johannes W 1978 Pressure comparing experiments with $\mathrm{NaCl}, \mathrm{AgCl}$, talc, and pyrophyllite assemblies in piston-cylinder apparatus; Neues Jahrb. Mineral., Monatsh. 284

Johannes W, Bell P M, Mao H K et al 1971 An interlaboratory comparison of piston-cylinder pressure calibration using the albite-break-down reaction; Contrib. Mineral. Petrol. 321

Mirwald P M, Getting I C and Kennedy G C 1975 Low-friction cell for piston-cylinder apparatus; J. Geophys. Res. 801519

Sirota N N, Mazurenko A M and Strukov N A 1974 Device for establishing high pressure and high temperature. Pat. 3790322 (USA) - office. Gazette. 05.02.74, no. 1, MKUB 306 11/32, HKU 425/77, 425/406 